\title{
Water Soluble Fluorescent Carbon Nanodots from Biosource for Cells Imaging
}

\author{
Kumud Malika Tripathi, ${ }^{1}$ Tuan Sang Tran, ${ }^{1}$ Tran Thanh Tung, \\ Dusan Losic, ${ }^{2}$ and TaeYoung Kim ${ }^{1}$ \\ ${ }^{1}$ Department of Bionanotechnology, Gachon University, 1342 Seongnam-daero, Sujeong-gu, Seongnam-si, \\ Gyeonggi-do 461-701, Republic of Korea \\ ${ }^{2}$ School of Chemical Engineering, The University of Adelaide, 5005 North Terrace, Adelaide, SA, Australia \\ Correspondence should be addressed to TaeYoung Kim; taeykim@gachon.ac.kr
}

Received 19 September 2016; Accepted 28 November 2016; Published 4 January 2017

Academic Editor: Rajesh R. Naik

Copyright (C) 2017 Kumud Malika Tripathi et al. This is an open access article distributed under the Creative Commons Attribution License, which permits unrestricted use, distribution, and reproduction in any medium, provided the original work is properly cited.

Carbon nanodots (CNDs) derived from a green precursor, kidney beans, was synthesized with high yield via a facile pyrolysis technique. The CND material was easily modified through simple oxidative treatment with nitric acid, leading to a high density "self-passivated" water soluble form (wsCNDs). The synthesized wsCNDs have been extensively characterized by using various microscopic and spectroscopic techniques and were crystalline in nature. The highly carboxylated wsCNDs possessed tunablephotoluminescence emission behavior throughout the visible region of the spectrum, demonstrating their application for multicolor cellular imaging of HeLa cells. The tunable-photoluminescence properties of "self-passivated" wsCNDs make them a promising candidate as a probe in biological cell-imaging applications.

\section{Introduction}

Carbon nanodots (CNDs) are emerging as new class of carbon-based fluorescent nanomaterial for their substantial applications in wide areas, owing to numerous alluring properties [1,2], which include environmental friendliness, favorable biocompatibility, excellent chemical stability, broad excitation spectra, resistance to photo-bleaching, and ease of surface modification [2]. One of the most noteworthy topics of the fluorescent CNDs is their potential to overcome the shortcomings (such as toxicity, photo-bleaching, and photoblinking) of conventional semiconductor quantum dots and organic dyes, especially in biomedical applications [3, 4]. CNDs can be easily and quickly internalized into living cells and demonstrate almost negligible toxicity [5]. Their versatile tunable properties along with solution-processing technology endow CNDs for enormous potential applications in photo-catalysis [6], photo-detectors [7], water remediation [8], sensors [9], energy storage [10], lasers [11], photovoltaic devices [7], LEDs [12], drug delivery [13], and bioimaging [14-16]. CNDs exhibit strong quantum effects due to their quantum size and quantum confinement, consequently exhibiting intriguing optical properties [17]. Furthermore, the controllable band gap and hence the fluorescent properties of CNDs can be artificially engineered by tuning their size and surface functionalities [18-20]. CNDs are a collection of hexagonal lattice of $\mathrm{sp}^{2}$ carbons incorporated with $\mathrm{sp}^{3}$ carbons and heteroatomic functional groups at a nanometer scale [17]. The large amounts of $\mathrm{C}-\mathrm{C}$ bonding and $\pi$-electronic conjugations that exist in CNDs are irregular and disrupted into small fragments [21]. Hence, CNDs could also be termed as highly defected composites of coexisting regions of aliphatic and aromatic carbons, which are usually modified with surface polar groups. CNDs exhibit excellent photostability, biocompatibility, and innocuousness properties in contrast with other allotropes of nanocarbons [17]. In addition, compared to other carbon nanomaterials (carbon nanotubes, graphenes, fullerenes, nanodiamonds, and carbon nanohorns), CNDs can be easily synthesized by relatively simpler and faster methods from a wide variety of precursors, without the need for any expensive and sophisticated instruments $[2,19]$. In consequence, CNDs 
offer significant potential to be used as an alternative to other carbon nanomaterials.

High aspect ratio, defects, and surface functionalities govern the properties of CNDs, which strongly depends upon the precursor material and synthetic conditions [21]. Cost and availability of precursor material are the prime concerns for commercial production of CNDs with easiness in synthesis. Top-down and bottom-up strategies are adapted for the synthesis of CNDs but complex, extreme synthetic conditions and the use of expensive energy-consuming devices are the major barriers hindering the use of these techniques. Thousands of raw materials that vary from laboratoryproduced chemicals and waste materials to biomass have been explored for the synthesis of CNDs [2, 19, 22]. In recent years, synthesis of fluorescent CNDs from biomass and waste materials in a simple and economical manner is of great interest. Among them, CNDs synthesized by using biomass as natural carbon precursors have been attracting tremendous interest in bioimaging and biomedical applications owing to their excellent biocompatibility. A facile synthesis of aqueous stable CNDs having high quantum yield is a current need of time. In the course with this quest, although many natural precursors and waste material have already been adapted for the synthesis of CNDs such as potato [23], orange waste peel [24], waste frying oil [22], lemon peel [6], almond husk [25], rice [26], and many more, however, kidney bean still remains to be explored despite being a rich source of protein, trace minerals, and good source of cholesterol-lowering fiber functionality [27]. Kidney beans meet the aforementioned requirements of a green precursor, owing to their global availability at low cost with well-defined variable properties.

Herein, we present a simple, facile, and economic approach for the synthesis of multicolor fluorescent water soluble carbon nanodots (wsCNDs) based on pyrolytic carbonization of precursor kidney beans. Oxidation of carbon soot introduces aqueous solubility and strong fluorescence emissions through the visible region of spectrum. Such synthesized wsCNDs are in the range of $10-30 \mathrm{~nm}$ in particle size with good aqueous solubility, stability in solution, and high photostability. The tunable emissive properties and fine aqueous solubility of these wsCNDs will hold promising advancements in biological imaging and constructing biosensors from an economic viewpoint.

\section{Experimental}

2.1. Materials. Kidney beans were purchased from a domestic shop in South Korea. Quinine sulfate was purchased from Sigma Aldrich. Nitric acid was obtained from Samchun Chemicals, Korea. $\mathrm{NaCl}, \mathrm{KI}$ and $\mathrm{Na}_{2} \mathrm{SO}_{4}$ were obtained from Alfa Aesar. All the chemicals were of analytical grade and were used as obtained. Deionized (DI) water was used throughout the experiment.

2.2. Instrumentation. Transmission electron microscopy (TEM) and high resolution TEM (HR-TEM) studies for the morphological characterization of wsCNDs were carried out with a Tecnai G2 at a $200 \mathrm{kV}$ accelerating voltage. Samples for TEM measurements were prepared by placing droplets of an aqueous solution of wsCNDs onto a carbon-coated copper grid and then dried under vacuum at room temperature overnight. The optical data of the wsCNDs was measured in quartz cells. Photoluminescence measurements were performed with a Varian fluorescence spectrometer in aqueous solution at room temperature. Fourier transform infrared (FTIR) spectra were recorded in solid state on a Jasco FT/IR-4100 spectrometer in the range of $400-4000 \mathrm{~cm}^{-1}$. Fluorescence imaging of wsCNDs under green and red band pass filter was done with a DM 2500 inverted microscope (Leica, Heerbrugg, Switzerland). Raman spectra were characterized with Renishaw with an excitation laser source of $514 \mathrm{~nm}$ under ambient conditions. The ultraviolet-visible (UV-Vis) absorption spectra of wsCNDs were recorded on a Varian 50 Bio UV-Vis spectrophotometer in aqueous solution at ambient conditions. The crystalline phases of CNDs and wsCNDs were identified using a Rigaku model $\mathrm{D}$ with monochromated $\mathrm{CuK}_{\alpha}$ radiation from 10 to $80^{\circ}$. Surface charge analysis was performed with zeta potentials measurements on a zeta potential analyzer ver. 5.72 (Brookhaven instruments). Thermogravimetric analysis (TGA) was carried out under continuous flow of nitrogen with a NETZSCH STA 409 PC/PG at a heating rate of $10^{\circ} \mathrm{C} \mathrm{m^{-1 }}$, starting from room temperature up to $1000^{\circ} \mathrm{C}$.

2.3. Synthesis of Water Soluble Carbon Nanodots (wsCNDs). CNDs were synthesized by the pyrolysis of kidney beans as a green carbon precursor in oxygen depleted environment. Prior to pyrolysis experiment, kidney beans were washed several times with DI water to remove any contaminations and dried in oven at $100^{\circ} \mathrm{C}$ overnight. Dried kidney beans were finely powdered in a mortar pestle. In a typical procedure, $5 \mathrm{~g}$ finely powdered kidney beans were taken in a capped quartz boat and heated at $450^{\circ} \mathrm{C}$ for $2 \mathrm{~h}$ in a furnace under an oxygen depleted environment at $5^{\circ} \mathrm{C} \mathrm{min}^{-1}$ heating rate. The furnace was then allowed to cool down naturally to room temperature and solid carbonaceous CNDs were collected. As-synthesized CNDs were hydrophobic in nature and insoluble in most organic solvents and water.

Aqueous solubility of synthesized CNDs was achieved by further oxidation with nitric acid as demonstrated in Figure 1 following earlier reports by Sonkar and coworkers [28]. Typically, $2 \mathrm{~g}$ of CNDs was refluxed in concentrated nitric acid $(100 \mathrm{~mL})$ for $10 \mathrm{~h}$ followed by centrifugation of the resulting solution for acid removal and then evaporation on a water bath until a slurry was formed and $\mathrm{pH}=7$ reached. This slurry was dried at room temperature to obtain wsCNDs. The yield of water soluble fluorescent wsCNDs was $\sim 84 \%$.

2.4. Procedures for the Imaging of HeLa Cells. HeLa cells (human cervical cancer cell line) were grown according to previously reported protocols [29]. In a typical procedure, HeLa cells were cultured in $10 \%(\mathrm{v} / \mathrm{v})$ fetal bovine serum and $4 \times 10^{-3} \mathrm{M} \mathrm{L}^{-1}$ glutamine in Dulbecco's modified Eagle medium (DMEM) in a humidified incubator containing 5\% $\mathrm{CO}_{2}$ at $37^{\circ} \mathrm{C}$ [29]. An aqueous solution of wsCNDs at a concentration of $0.25 \mathrm{mg} \mathrm{mL}^{-1}$ was added to the culture medium. After incubation for $4 \mathrm{~h}$, HeLa cells were fixed with $4 \mathrm{wt} \%$ para-formaldehyde and washed thoroughly four times 


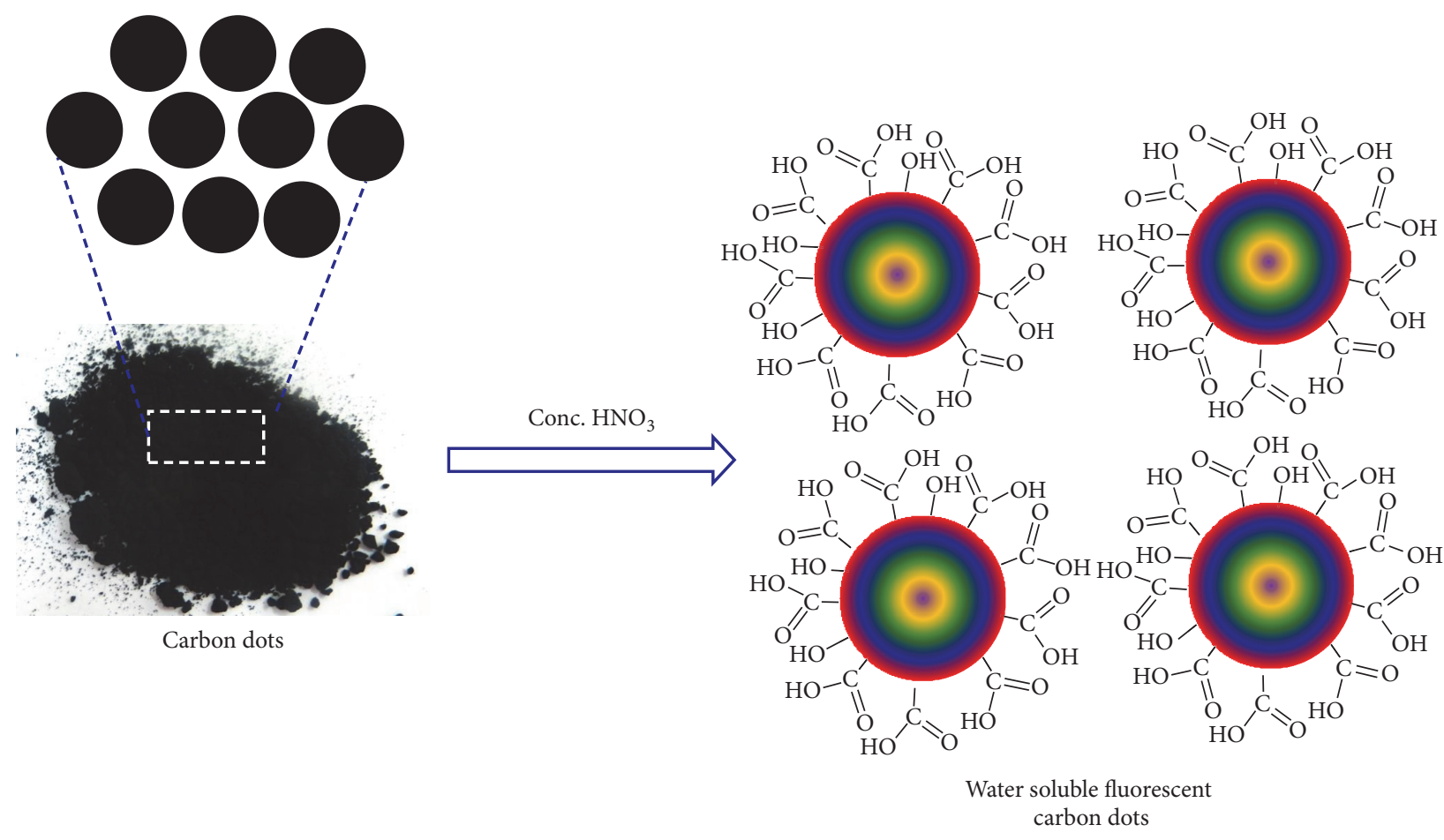

FIGURE 1: A schematic illustration for the synthesis of wsCNDs from kidney bean.

(1.0 mL each) with pH 7.4 PBS buffer solution to remove free wsCNDs and nonspecific adsorption on the cell membrane. The fluorescence imaging of wsCNDs tagged HeLa cells was done with a Leica inverted microscope under 488 and $532 \mathrm{~nm}$ band pass filter.

\section{Results and Discussion}

CNDs were effectively synthesized via pyrolysis of kidney bean as green precursor. Water solubility in CNDs was achieved by simple oxidative treatment with conc. nitric acid as shown in Figure 1. Oxidation with conc. nitric acid generated high density hydrophilic carboxylic acid and hydroxyl groups on wsCNDs surface and therefore introduces aqueous solubility along with photoluminescent properties.

3.1. Characterization of Carbon Nanodots (CNDs). The morphology and microstructure of wsCNDs was characterized with TEM and HRTEM. wsCNDs are spherical in nature and not uniform in size as shown in Figure 2(a). The size distribution of wsCNDs was performed via the statistical analysis of more than 100 particles. wsCNDs have an overall size distribution from 10 to $50 \mathrm{~nm}$, whereby most of the particles lie in the range of $20-30 \mathrm{~nm}$ as shown in Figure 2(b). This inhomogeneous size may be attributed to the tunable multicolor fluorescence of the wsCNDs due to the quantum-confinement effect and characteristics for spherical nanocarbons [30]. High resolution TEM image as shown in Figure 2(c) reveals the spherical size of wsCNDs. Crystalline structure of wsCNDs was confirmed by HRTEM analysis (Figure 2(d)). wsCNDs exhibited lattice spacing of $0.26 \mathrm{~nm}$, attributed to the (002) facet of graphite (Figure 2(d)).
FTIR analysis was performed to obtain structural insight about the surface functionalization of wsCNDs. Surface functionalization of wsCNDs due to the extensive oxidation is verified by the presence of characteristic $-\mathrm{C}=\mathrm{C},-\mathrm{C}=\mathrm{O}$, and $-\mathrm{O}-\mathrm{H}$ stretching bands in FTIR spectrum. As shown in Figure 3(a), the broad absorption $\sim 3387 \mathrm{~cm}^{-1}$ is assigned to $-\mathrm{O}-\mathrm{H}$ stretching of $-\mathrm{COOH}$ groups. A peak at $2935 \mathrm{~cm}^{-1}$ is attributed to $-\mathrm{CH}_{2}$ vibrations. The sharp band at $1588 \mathrm{~cm}^{-1}$ was assigned to the vibrational absorption band of $\mathrm{C}=\mathrm{C}$, while band $\sim 1713 \mathrm{~cm}^{-1}$ was attributed to the presence of $\mathrm{C}=\mathrm{O}$. The peak around $1214 \mathrm{~cm}^{-1}$ confirms the $-\mathrm{C}-\mathrm{O}$ stretching of graphitic carbons [25]. FTIR results confirm the presence of an abundant number of surfacial hydroxyl and carboxyl groups on wsCNDs and, therefore, the hydrophilic nature of wsCNDs. Further investigation of surface defects and degree of structural disorder in graphitic carbon domains was elaborated with Raman spectroscopic technique, which proved quite significant $[31,32]$. Raman spectra of CNDs and wsCNDs exhibited two broad peaks at $1343 \mathrm{~cm}^{-1}, 1588 \mathrm{~cm}^{-1}$ (wsCNDs) and $1352 \mathrm{~cm}^{-1}, 1593 \mathrm{~cm}^{-1}$ (CNDs), attributed to the $D$ ( $\mathrm{sp}^{3}$-hybridized) and $G$ ( $\mathrm{sp}^{2}$-hybridized) bands, respectively, as demonstrated in Figure $3(\mathrm{~b})$. The $D$ band is attributed to the lattice defects in the graphite plane and represents vibrations of carbon atoms with dangling bonds. The $G$ band corresponds to the $\mathrm{E}_{2} \mathrm{~g}$ mode of graphite and is associated with in-plane vibrations of $\mathrm{sp}^{2}$-hybridized carbon atoms $[33,34] . I_{D} / I_{G}$ is a reliable indicator to verify the degree of disorder in the graphitic pool of CNDs into defective forms of $\mathrm{sp}^{3}$ configuration. Higher $I_{D} / I_{G}$ values (1.3) (which is a characteristic of the disorder extent) in contrast to asprepared $\mathrm{CDs}\left(I_{D} / I_{G}=1.05\right)$ reveal the higher degree of 


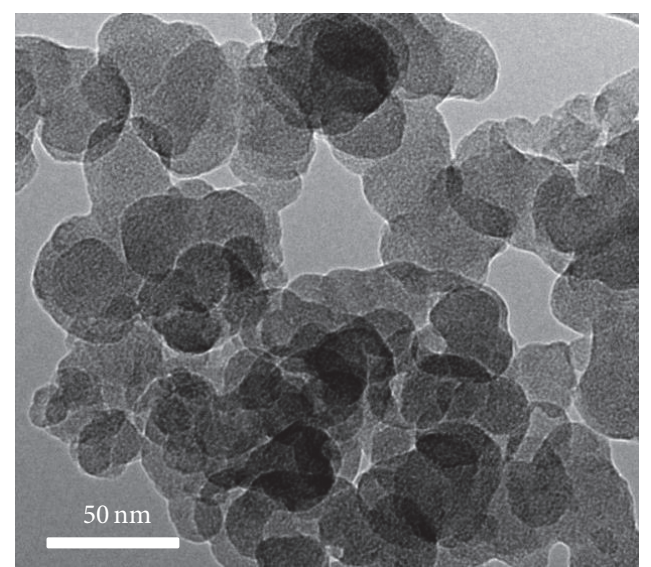

(a)

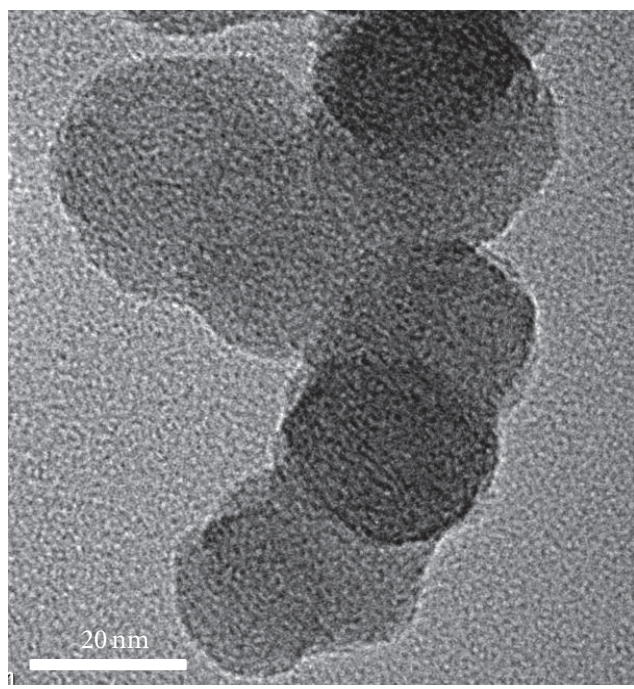

(c)

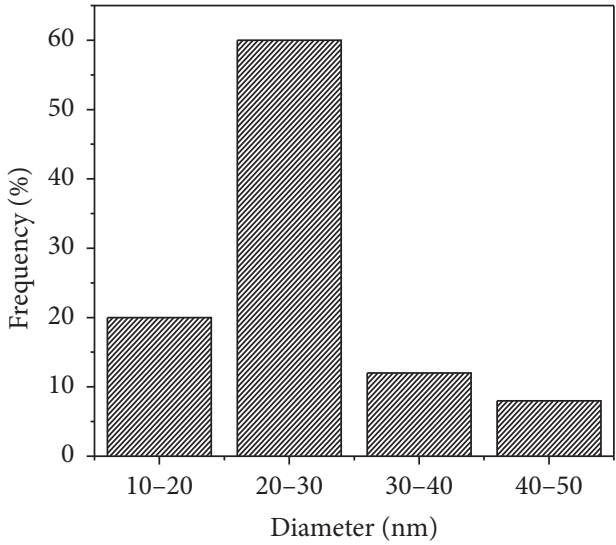

(b)

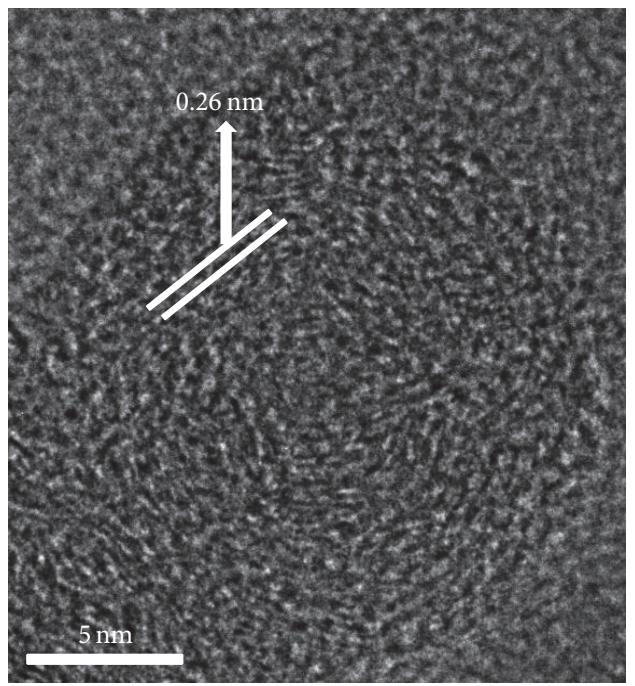

(d)

FIGURE 2: (a) Low resolution TEM image of wsCNDs; (b) size distribution histogram of wsCNDs; (c) high resolution TEM image of wsCNDs; (d) HRTEM image of wsCNDs showing interlayer spacing.

defects and are attributed to higher quantum yield values without any surface passivation in wsCNDs.

The X-ray powder diffraction (XRD) patterns were used to investigate the crystallinity of the synthesized wsCNDs. XRD patterns of the CNDs and wsCNDs display a slightly

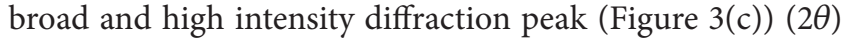
centered at $23.8^{\circ}$ owing to graphitic (002) lattice spacing, which arises due to the presence of highly disordered $\left(\mathrm{sp}^{3}\right)$ carbon atoms and is consistent with HRTEM results [35, 36]. Thermal gravimetric analysis (TGA) was employed to investigate the thermal stability of wsCNDs at a heating rate of $10^{\circ} \mathrm{C} \mathrm{min}{ }^{-1}$ under a nitrogen atmosphere. The comparative TGA analysis for CNDs (black line) and wsCNDs (blue line) is displayed in Figure 3(d). CNDs exhibited higher thermal stability than wsCNDs and lose $31.84 \%$ of their total weight at $1000^{\circ} \mathrm{C}$, presumably due to the thermal desorption of moisture and trace amounts of $\mathrm{C}-\mathrm{H}$ and $\mathrm{C}-\mathrm{O}$ functional groups [37]. Weight losses in wsCNDs were more prominent, exhibiting $\sim 54.05 \%$ weight loss at $1000^{\circ} \mathrm{C}$. The higher weight loss in wsCNDs was attributed to the degradation of highly dense oxygenated functional groups introduced during the oxidative process of wsCNDs via the destruction of the surfacial graphitic framework [9]. A highly negative apparent zeta potential value of wsCNDs in aqueous solution at $\mathrm{pH}$ $=7$ was $-43.80 \mathrm{mV}$, substantiating the existence of high density negative surface functionalities. Raman spectra, FTIR spectra, TGA, and zeta potential value confirm the high degree of carboxylic acid functionalization in wsCNDs.

3.2. Absorbance, Photoluminescence (PL) Emission, Excitation, and Fluorescence Imaging Studies. Absorption spectrum of wsCNDs is shown in Figure 4(a). wsCNDs exhibited a broad spectrum, with continual increase from 800 to $200 \mathrm{~nm}$ attributed to the $\pi-\pi^{*}$ and $n-\pi^{*}$ transitions of the $\mathrm{C}=\mathrm{C}$ and $-\mathrm{C}=\mathrm{O}$ bonds, respectively. UV-Vis spectrum indicates the absence of any other morphology of nanocarbons or 


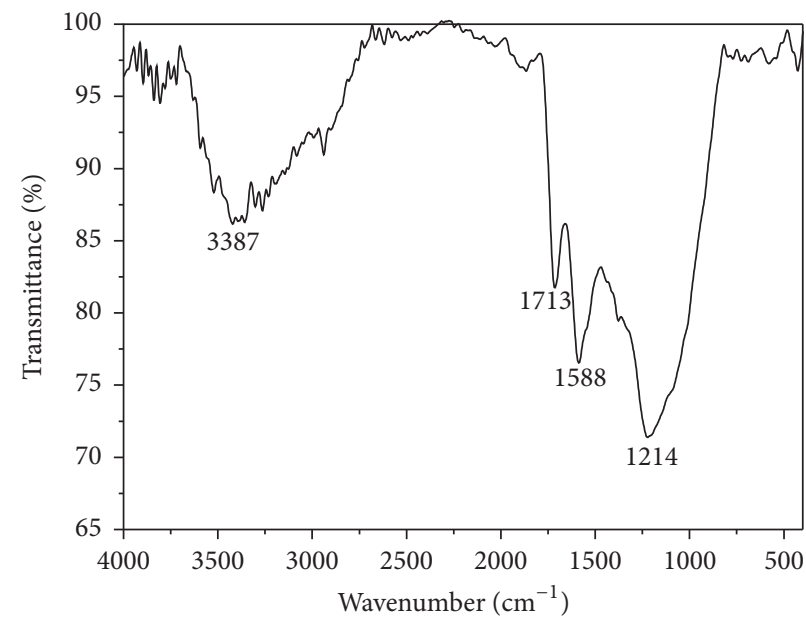

(a)

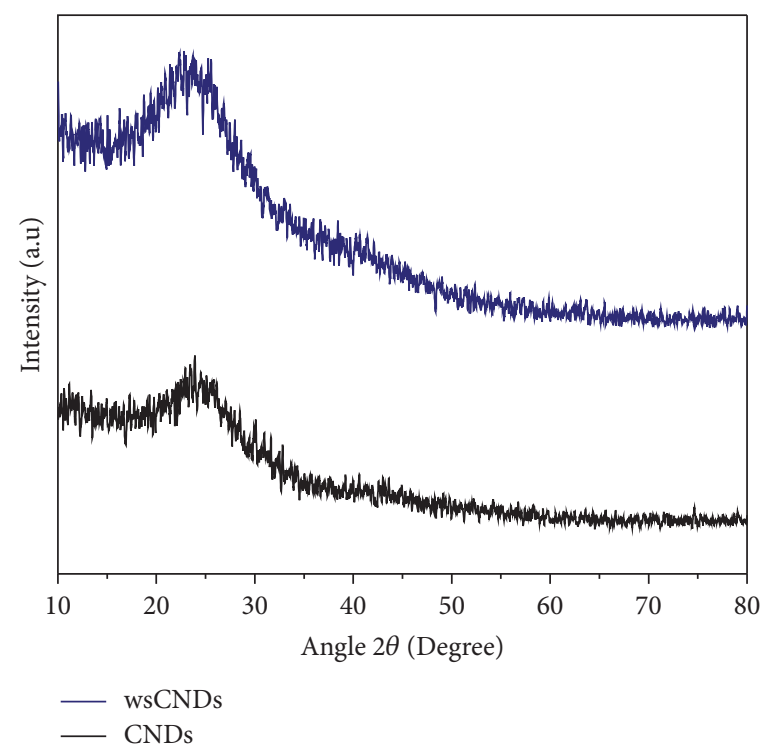

(c)

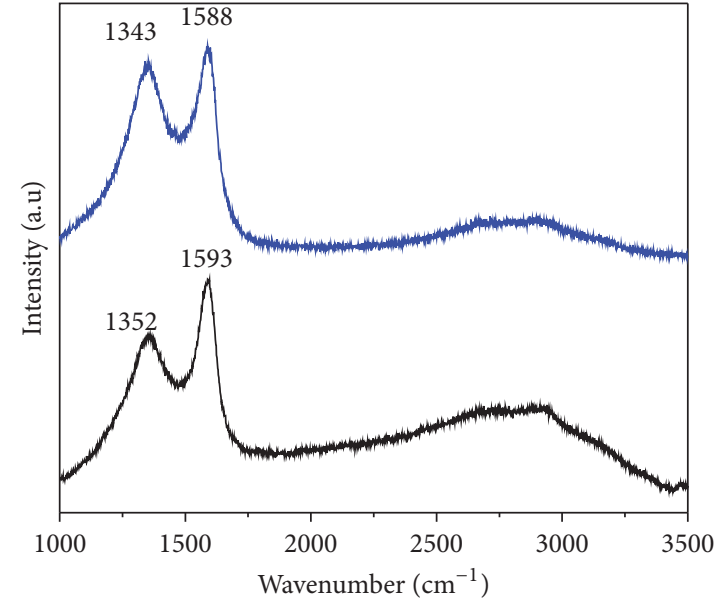

- wsCNDs

- CNDs

(b)

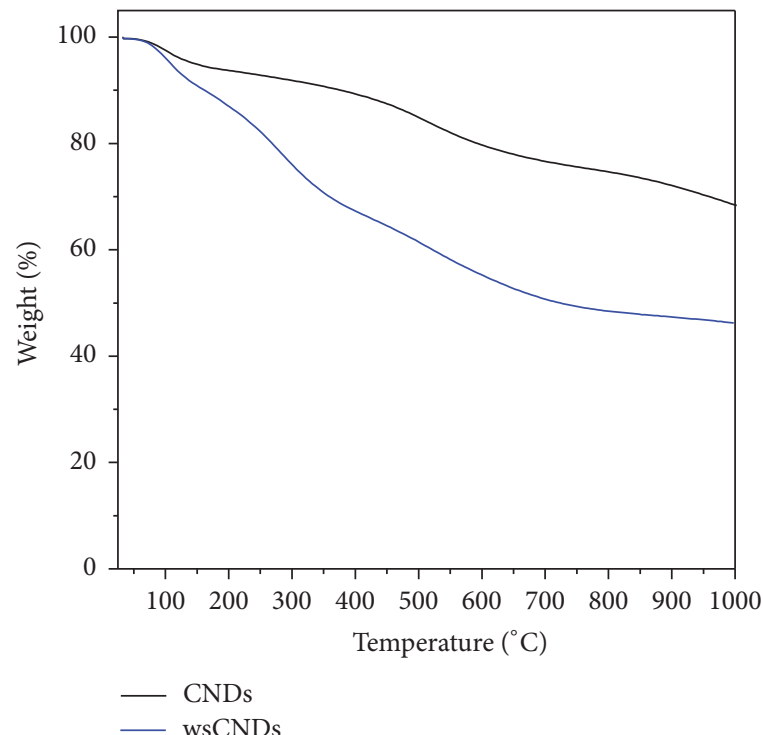

(d)

FIGURE 3: (a) FTIR spectrum of wsCNDs. (b) Raman and (c) XRD spectra of wsCNDs (blue line) and CNDs (black line); (d) thermal gravimetric analysis (TGA) of CNDs (black line) and wsCNDs (blue line).

amorphous carbon, as resulting from partial carbonization of precursor, since there is no sign of background absorbance in the visible region [35]. Aqueous solution of wsCNDs after several weeks of storage at room temperature did not result in any visible aggregation, demonstrating the excellent stability of wsCNDs in solution. A detailed PL study with different excitation wavelengths $\left(\lambda_{\text {ex }}\right)$ ranging from 340 to $620 \mathrm{~nm}$ was analyzed to explore the optical properties of wsCNDs. The emission spectrum of wsCNDs is excitation dependent as shown in Figure 4(b) and is characterized as a generic feature of nanoparticles possessing carbogenic core [38]. When excitation wavelength changes from 340 to $620 \mathrm{~nm}$ on a continuous increase of $20 \mathrm{~nm}$, emission wavelength red-shifted. The PL emission of wsCNDs ranges throughout the visible region of spectrum covers $461-658 \mathrm{~nm}$ with maximum intensity at $461 \mathrm{~nm}$ centered peak at $340 \mathrm{~nm}$ excitation wavelength. The relationship between excitation wavelength and emission wavelength is shown in Figure 4(c). The excitation spectrum at $460 \mathrm{~nm}$ emission wavelength exhibited two bands at 332 and $393 \mathrm{~nm}$ (Figure 4(d)) which matches with core and surface absorption, respectively, and indicates the presence of various types of light emitting centers $[9,35]$. The fluorescence microscopic imaging reveals the highly florescence nature of wsCNDs under different band pass filters as shown in Figures 4(e) and 4(f). wsCNDs exhibited strong green and red emissions in the visible region at 448 and $561 \mathrm{~nm}$ band pass filters.

The origin of PL emissions in CNDs still remains a big mystery and is highly controversial with different explanations due to various synthetic approaches and numerous 

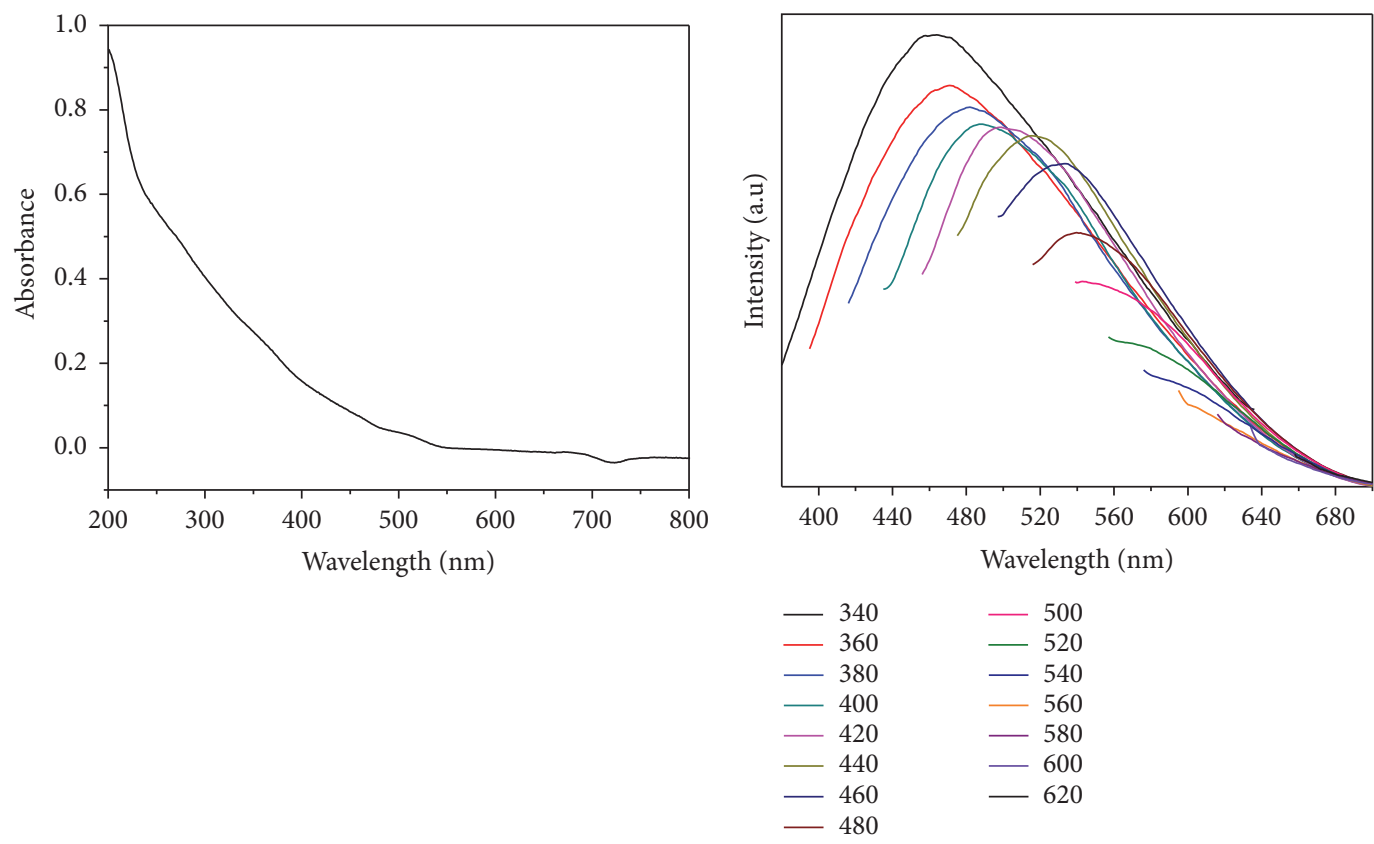

(a)

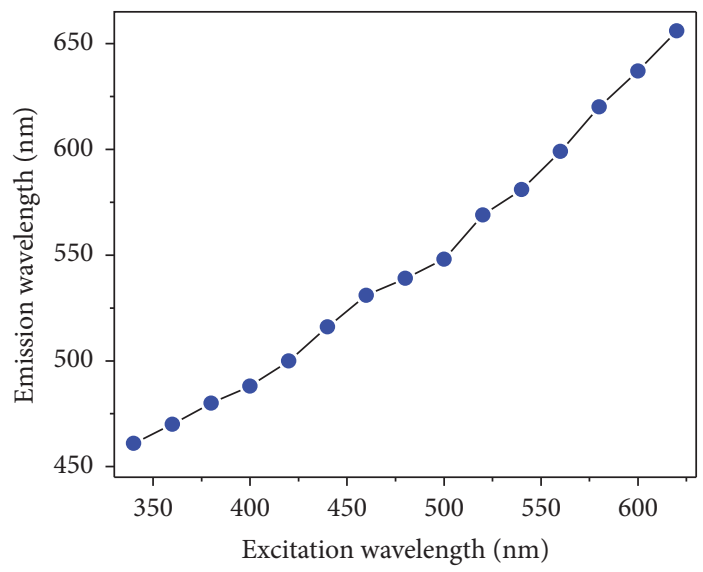

(c)

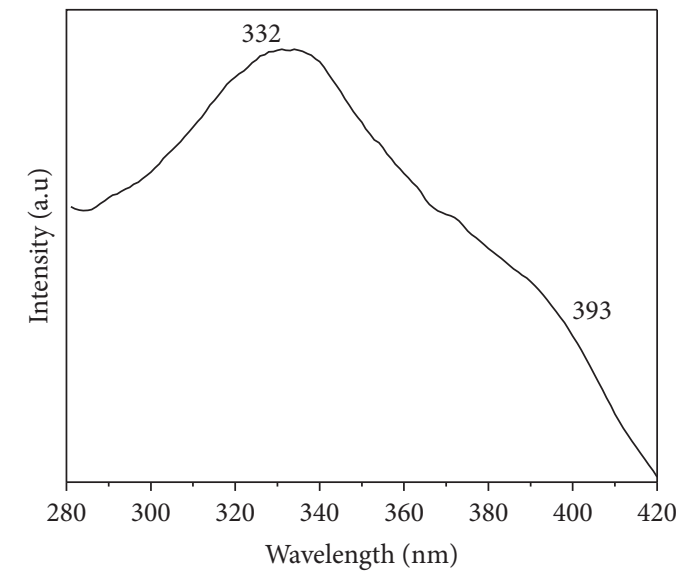

(d)
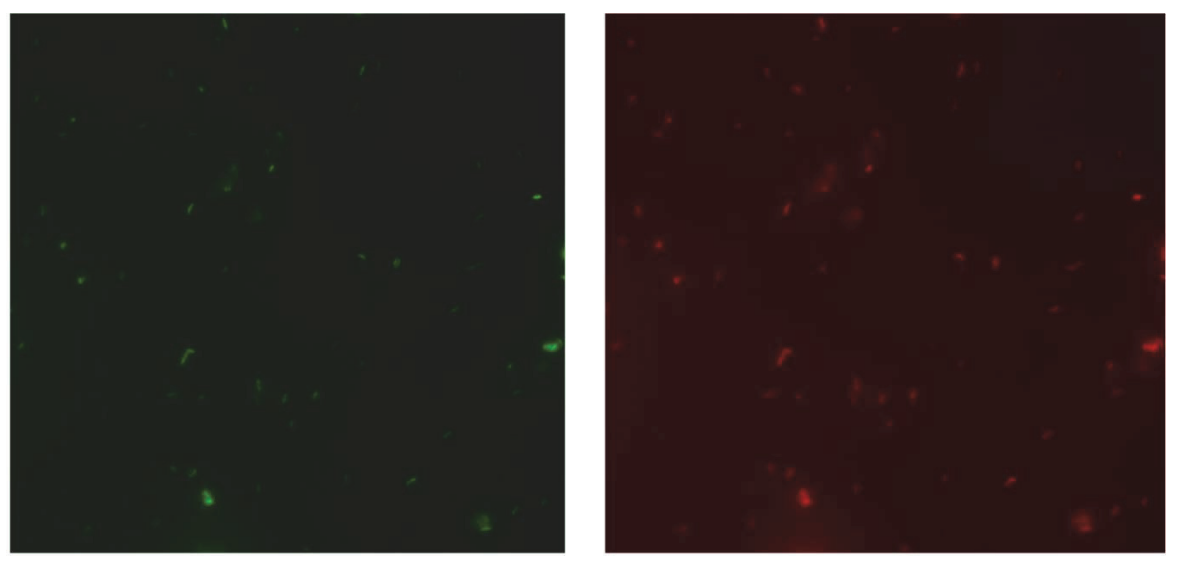

(e)

(f)

FIGURE 4: (a) Absorption spectra of wsCNDs; (b) tunable emission spectra of wsCNDs in the range of 340-620 nm excitation wavelength on a continuous increment of $20 \mathrm{~nm}$; (c) variation in emissions center with excitation, $\lambda_{\mathrm{em}}$ was efficiently showing a bathochromic shift when move from one $\lambda_{\mathrm{ex}}$ to the next with $20 \mathrm{~nm}$ increment to track the shift in the PL spectrum with $\lambda_{\text {ex }}$, the data points were gets from panel (b); (d) photoluminescent excitation spectrum on $460 \mathrm{~nm}$ emission wavelength. Optical images of wsCNDs at (e) $488 \mathrm{~nm}$ (green) and (f) $561 \mathrm{~nm}$ (red) band pass filters, respectively. 


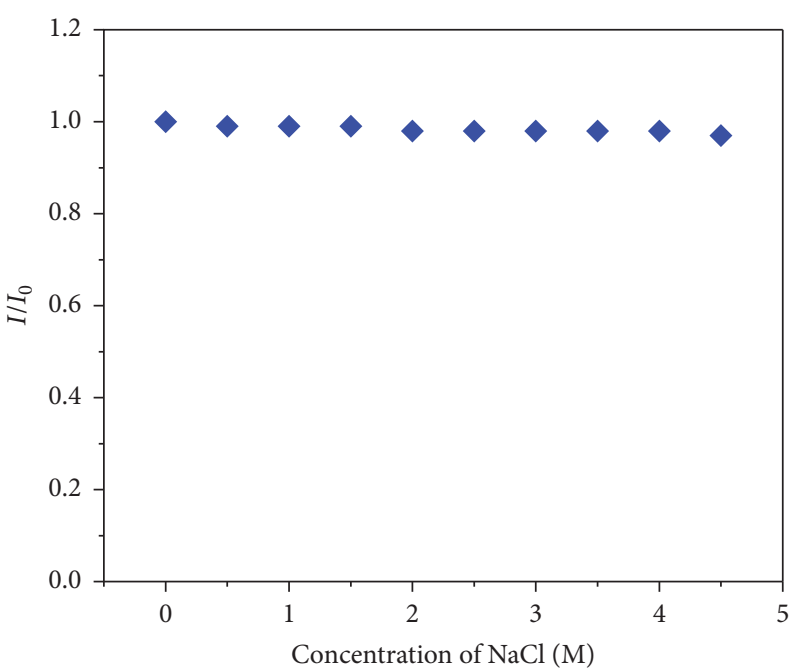

(a)

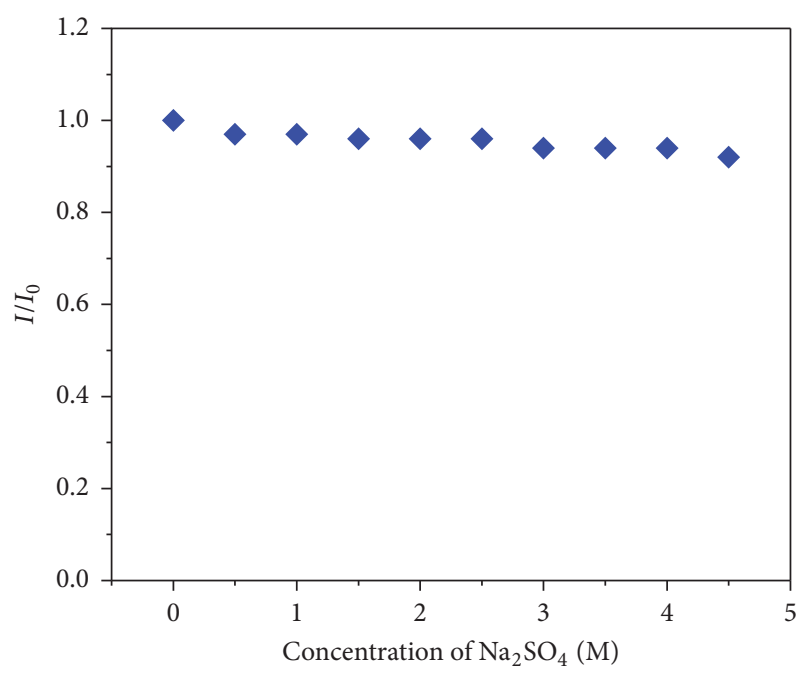

(c)

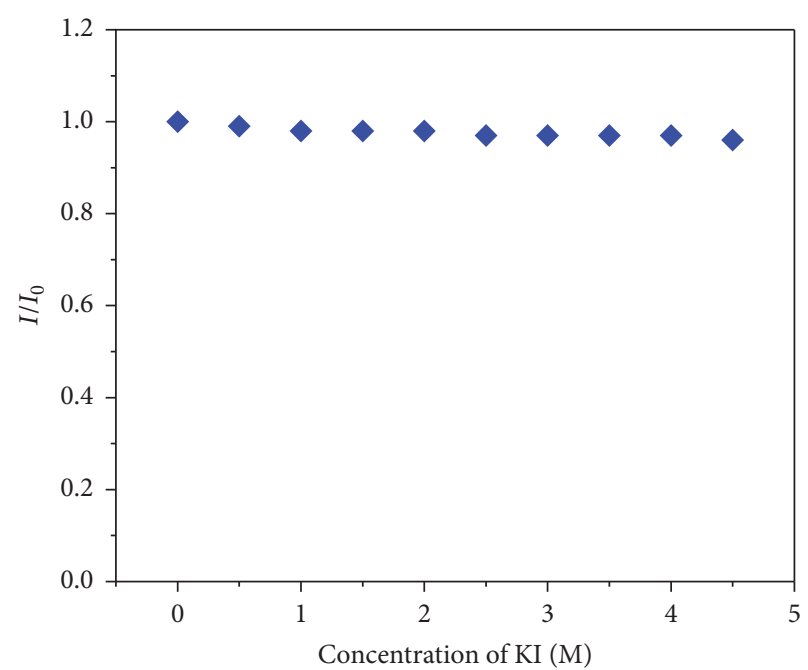

(b)

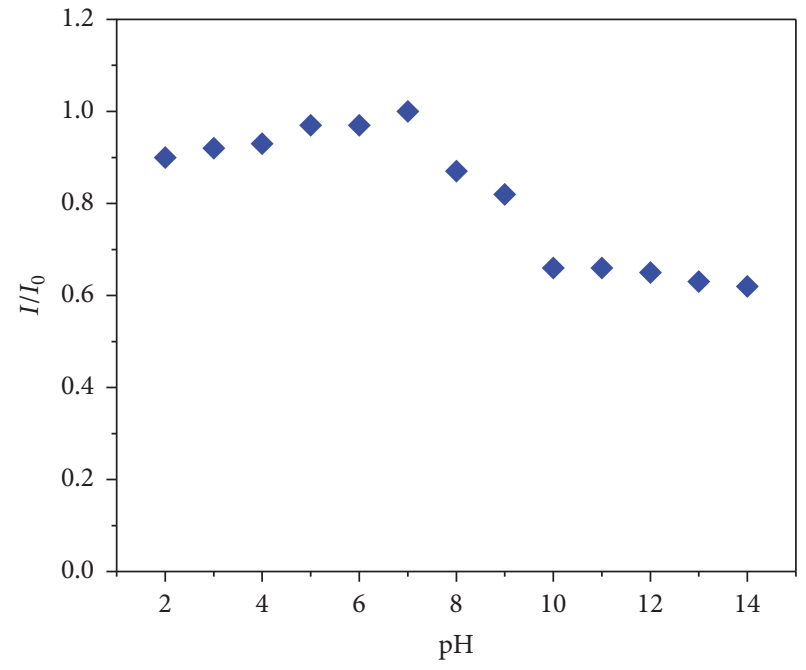

(d)

FIGURE 5: Photostability test of wsCNDs in the presence of a high ionic strength of (a) sodium chloride; (b) potassium iodide; and (c) sodium sulfate. (d) Effect of $\mathrm{pH}$ on fluorescence emission intensity of wsCNDs.

precursor ingredients along with multiple complicated components and structures. Tunable emissions of wsCNDs reflect the effect from different surface emissive traps and particle size. Previous reports indicated that the polar surface functionalities such as hydroxyl and carboxylic groups dominated the "giant red-edge effect" and hence attributed the excitation dependent emission behavior of CNDs [39]. Zhang and coworkers proposed that oxygenous functional groups lead to the defects and furthermore their respective energy level having both localized and delocalized molecular orbitals in the band gap leading to tunable emissions in the wide region of spectra [40]. In another report by Demchenko and Dekaliuk, CND was proposed as an assembly of integral quantum emitters (anisotropic arrangements of aromatic and aliphatic carbon domains leading to generation of excitonic H-type aggregates) and tunable emissions attributed to summation of their individual contributions [21].
The quantum yield of wsCNDs was measured to be $8 \%$ with reference to Quinine sulfate, indicating the strong quantum confinement of particles. No perceptible change in fluorescence emission intensity was observed after 3 hours under continuous irradiation of UV light. Significantly, the aqueous solution of wsCNDs exhibited good photostability, that is, good luminescence properties, and their appearance remains unaffected, when stored in closed vessels in an inert atmosphere without any distinct reduction in fluorescence intensity. We have also studied the effect of ionic strength and $\mathrm{pH}$ variations on $\mathrm{PL}$ emission of wsCNDs to understand better the photostability as presented in Figure 5. No much changes in PL emissions were observed in presence of high ionic strength of $\mathrm{NaCl}, \mathrm{KI}$, and $\mathrm{Na}_{2} \mathrm{SO}_{4}$ as shown in Figures $5(\mathrm{a})-5(\mathrm{c})$. Hence, wsCNDs could potentially be used as fluorescent probe at various physical salt concentrations. However, significant change in PL intensity was observed 


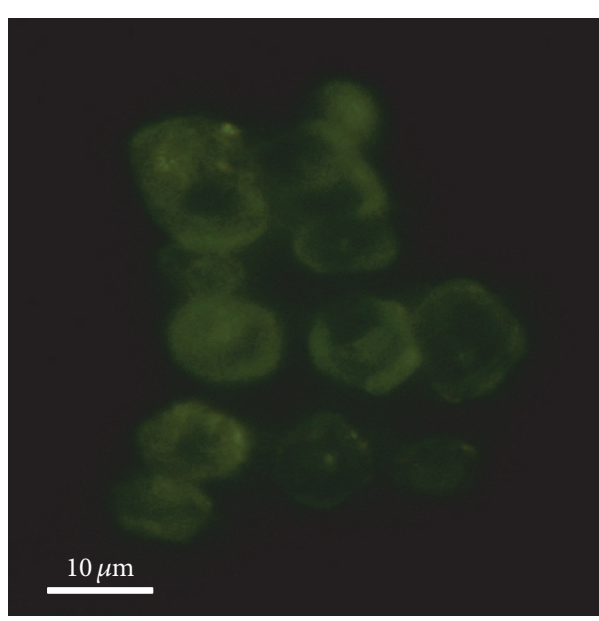

(a)

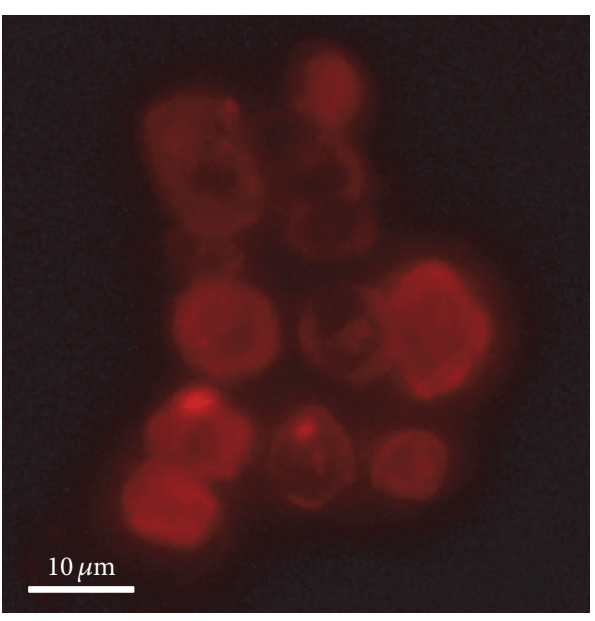

(b)

FIgURE 6: Fluorescence microscopy images of HeLa cells (a) under $488 \mathrm{~nm}$ (green) and (b) $561 \mathrm{~nm}$ (red) band pass filters, respectively.

with varied $\mathrm{pH}$ from 2 to 14 due to protonation and deprotonation of surfacial carboxylic groups of wsCNDs in acidic and basic $\mathrm{pH}$, respectively [41]. PL intensity decreases with change in $\mathrm{pH}$ and effect is more pronounced in basic conditions as shown in Figure 5(d).

3.3. Imaging of HeLa Cells. Potential application of wsCNDs was validated as a fluorescent probe for the multicolor imaging of HeLa cells owing to facile synthetic procedure and intriguingly strong PL properties. After incubation with wsCNDs in culture medium for $4 \mathrm{~h}$ cells were washed with PBS buffer to remove free wsCNDs and imaged under a fluorescence microscope. HeLa cells exhibited bright green and red fluorescence under band pass filters of 488 and $561 \mathrm{~nm}$ (Figure 6). A strong fluorescence on the cytoplasmic area and cell membrane of HeLa cells in Figures 6(a) and 6(b) show that wsCNDs were distributed perinuclearly, thus demonstrating a good degree of cellpermeability of the wsCNDs into HeLa cells [42]. It also indicated that wsCNDs remained fluorescent in the cellular environment.

\section{Conclusions}

A simple and easily scale up method of carbonization of kidney beans provided an economic, environmental friendly approach for the quantitative synthesis of CNDs without accompaniment of any amorphous carbon. The simple treatment of CNDs with conc. nitric acid imparted desirable negative surface functionalities and multicolor fluorescence with a quantum yield of $8 \%$. wsCNDs showed good stability and are highly fluorescent in nature with tunable emission behavior. The materials show excellent photostability even under high ionic strength environment. By demonstrating a good degree of cell-permeability of the wsCNDs into HeLa cells, this material can be explored for cellular imaging applications and promisingly in biosensing and drug delivery applications.

\section{Competing Interests}

The authors declare that they have no competing interests.

\section{Acknowledgments}

The authors would like to acknowledge the financial support from the R\&D Convergence Program of MSIP (Ministry of Science, ICT and Future Planning) and NST (National Research Council of Science \& Technology) of Republic Korea (Grant CAP-13-02-ETRI). This research was also supported by Nano Material Technology Development Program through the National Research Foundation of Korea (NRF) funded by the Ministry of Science, ICT and Future Planning (NRF-2016M3A7B4027712). The authors thank the support of the Australian Research Council (IH150100003).

\section{References}

[1] P. G. Luo, S. Sahu, S.-T. Yang et al., "Carbon "quantum” dots for optical bioimaging," Journal of Materials Chemistry B, vol. 1, no. 16, pp. 2116-2127, 2013.

[2] S. Y. Lim, W. Shen, and Z. Gao, "Carbon quantum dots and their applications," Chemical Society Reviews, vol. 44, no. 1, pp. 362381, 2015.

[3] G. Hong, S. Diao, A. L. Antaris, and H. Dai, "Carbon nanomaterials for biological imaging and nanomedicinal therapy," Chemical Reviews, vol. 115, no. 19, pp. 10816-10906, 2015.

[4] S. Y. Choi, J. P. Shim, D. S. Kim, T. Kim, and K. S. Suh, "Aqueous synthesis of CdTe quantum dot using dithiol-functionalized ionic liquid," Journal of Nanomaterials, vol. 2012, Article ID 519458, 6 pages, 2012.

[5] A. Zhao, Z. Chen, C. Zhao, N. Gao, J. Ren, and X. Qu, "Recent advances in bioapplications of C-dots," Carbon, vol. 85, pp. 309327, 2015.

[6] A. Tyagi, K. M. Tripathi, N. Singh, S. Choudhary, and R. K. Gupta, "Green synthesis of carbon quantum dots from lemon peel waste: applications in sensing and photocatalysis," RSC Adv. vol. 6, no. 76, pp. 72423-72432, 2016. 
[7] C. Xie, B. Nie, L. Zeng et al., "Core-shell heterojunction of silicon nanowire arrays and carbon quantum dots for photovoltaic devices and self-driven photodetectors," ACS Nano, vol. 8, no. 4, pp. 4015-4022, 2014.

[8] K. M. Tripathi and N. R. Gupta, "Nano-carbons from pollutant soot: a cleaner approach toward clean environment," in Smart Materials for Waste Water Applications, pp. 127-154, John Wiley \& Sons, 2016

[9] K. M. Tripathi, A. Bhati, A. Singh et al., "From the traditional way of pyrolysis to tunable photoluminescent water soluble carbon nano-onions for cell imaging and selective sensing of glucose," RSC Adv., vol. 6, no. 44, pp. 37319-37329, 2016.

[10] A. Tyagi, K. M. Tripathi, and R. K. Gupta, "Recent progress in micro-scale energy storage devices and future aspects," Journal of Materials Chemistry A, vol. 3, no. 45, pp. 22507-22541, 2015.

[11] H. Zhu, W. Zhang, and S. F. Yu, "Realization of lasing emission from graphene quantum dots using titanium dioxide nanoparticles as light scatterers," Nanoscale, vol. 5, no. 5, pp. 1797-1802, 2013.

[12] X. Zhang, Y. Zhang, Y. Wang et al., "Color-switchable electroluminescence of carbon dot light-emitting diodes," ACS Nano, vol. 7, no. 12, pp. 11234-11241, 2013.

[13] H. Ding, F. Du, P. Liu, Z. Chen, and J. Shen, "DNA-carbon dots function as fluorescent vehicles for drug delivery," ACS Applied Materials \& Interfaces, vol. 7, no. 12, pp. 6889-6897, 2015.

[14] P. G. Luo, F. Yang, S.-T. Yang et al., "Carbon-based quantum dots for fluorescence imaging of cells and tissues," RSC Advances, vol. 4, no. 21, pp. 10791-10807, 2014.

[15] G. E. Lecroy, S. K. Sonkar, F. Yang et al., "Toward structurally defined carbon dots as ultracompact fluorescent probes," ACS Nano, vol. 8, no. 5, pp. 4522-4529, 2014.

[16] M. Ghosh, S. K. Sonkar, M. Saxena, and S. Sarkar, "Carbon nano-onions for imaging the life cycle of Drosophila melanogaster," Small, vol. 7, no. 22, pp. 3170-3177, 2011.

[17] L. Cao, M. J. Meziani, S. Sahu, and Y.-P. Sun, "Photoluminescence properties of graphene versus other carbon nanomaterials," Accounts of Chemical Research, vol. 46, no. 1, pp. 171-180, 2013.

[18] H. Tetsuka, R. Asahi, A. Nagoya et al., "Optically tunable aminofunctionalized graphene quantum dots," Advanced Materials, vol. 24, no. 39, pp. 5333-5338, 2012.

[19] P.-C. Hsu, Z.-Y. Shih, C.-H. Lee, and H.-T. Chang, "Synthesis and analytical applications of photoluminescent carbon nanodots," Green Chemistry, vol. 14, no. 4, pp. 917-920, 2012.

[20] K. M. Tripathi, T. Kim, D. Losic, and T. T. Tung, "Recent advances in engineered graphene and composites for detection of volatile organic compounds (VOCs) and non-invasive diseases diagnosis," Carbon, vol. 110, pp. 97-129, 2016.

[21] A. P. Demchenko and M. O. Dekaliuk, "The origin of emissive states of carbon nanoparticles derived from ensemble-averaged and single-molecular studies," Nanoscale, vol. 8, no. 29, pp. 14057-14069, 2016.

[22] Y. Hu, J. Yang, J. Tian, L. Jia, and J.-S. Yu, "Waste frying oil as a precursor for one-step synthesis of sulfur-doped carbon dots with $\mathrm{pH}$-sensitive photoluminescence," Carbon, vol. 77, pp. 775782, 2014.

[23] K. Wang, F. Guan, H. Li, M. Li, H. Feng, and H. Fan, "Onestep synthesis of carbon nanodots for sensitive detection of cephalexin," RSC Advances, vol. 5, no. 26, pp. 20511-20515, 2015.

[24] A. Prasannan and T. Imae, "One-pot synthesis of fluorescent carbon dots from orange waste peels," Industrial \& Engineering Chemistry Research, vol. 52, no. 44, pp. 15673-15678, 2013.
[25] K. M. Tripathi, A. Tyagi, M. Ashfaq, and R. K. Gupta, “Temperature dependent, shape variant synthesis of photoluminescent and biocompatible carbon nanostructures from almond husk for applications in dye removal," RSC $A d v$., vol. 6, no. 35, pp. 29545-29553, 2016.

[26] S. K. Sonkar, M. Saxena, M. Saha, and S. Sarkar, "Carbon nanocubes and nanobricks from pyrolysis of rice," Journal of Nanoscience and Nanotechnology, vol. 10, no. 6, pp. 4064-4067, 2010.

[27] A. Pusztai, E. M. Clarke, T. P. King, and J. C. Stewart, "Nutritional evaluation of kidney beans (Phaseolus vulgaris): chemical composition, lectin content and nutritional value of selected cultivars," Journal of the Science of Food and Agriculture, vol. 30, no. 9, pp. 843-848, 1979.

[28] A. Bhati, A. Singh, K. M. Tripathi, and S. K. Sonkar, "Sunlightinduced photochemical degradation of methylene blue by water-soluble carbon nanorods," International Journal of Photoenergy, vol. 2016, Article ID 2583821, 8 pages, 2016.

[29] Y. Cui, C. Zhang, L. Sun, Z. Hu, and X. Liu, "Simple and efficient synthesis of strongly green fluorescent carbon dots with upconversion property for direct cell imaging," Particle \& Particle Systems Characterization, vol. 32, no. 5, pp. 542-546, 2015.

[30] V. Georgakilas, J. A. Perman, J. Tucek, and R. Zboril, “Broad family of carbon nanoallotropes: classification, chemistry, and applications of fullerenes, carbon dots, nanotubes, graphene, nanodiamonds, and combined superstructures," Chemical Reviews, vol. 115, no. 11, pp. 4744-4822, 2015.

[31] M. A. Pimenta, G. Dresselhaus, M. S. Dresselhaus, L. G. Cançado, A. Jorio, and R. Saito, "Studying disorder in graphitebased systems by Raman spectroscopy," Physical Chemistry Chemical Physics, vol. 9, no. 11, pp. 1276-1291, 2007.

[32] T. Kim, G. Jung, S. Yoo, K. S. Suh, and R. S. Ruoff, "Activated graphene-based carbons as supercapacitor electrodes with macro- and mesopores," ACS Nano, vol. 7, no. 8, pp. 68996905, 2013.

[33] T. S. Tran, S. J. Park, S. S. Yoo, T.-R. Lee, and T. Kim, "High shearinduced exfoliation of graphite into high quality graphene by Taylor-Couette flow," RSC Advances, vol. 6, no. 15, pp. $12003-$ 12008, 2016.

[34] T. Kim, H. Lee, J. Kim, and K. S. Suh, "Synthesis of phase transferable graphene sheets using ionic liquid polymers," ACS Nano, vol. 4, no. 3, pp. 1612-1618, 2010.

[35] V. Ramanan, S. K. Thiyagarajan, K. Raji, R. Suresh, R. Sekar, and P. Ramamurthy, "Outright green synthesis of fluorescent carbon dots from eutrophic algal blooms for in vitro imaging," ACS Sustainable Chemistry \& Engineering, vol. 4, no. 9, pp. 47244731, 2016.

[36] S. K. Sonkar, K. M. Tripathi, and S. Y. Sarkar, "Ferromagnetic behaviour of anthropogenic multi-walled carbon nanotubes trapped in spider web indoor," Journal of Nanoscience and Nanotechnology, vol. 14, no. 3, pp. 2532-2538, 2014.

[37] K. M. Tripathi, A. K. Sonker, A. Bhati et al., "Large-scale synthesis of soluble graphitic hollow carbon nanorods with tunable photoluminescence for the selective fluorescent detection of DNA," New Journal of Chemistry, vol. 40, no. 2, pp. 1571-1579, 2016.

[38] Y.-P. Sun, B. Zhou, Y. Lin et al., "Quantum-sized carbon dots for bright and colorful photoluminescence," Journal of the American Chemical Society, vol. 128, no. 24, pp. 7756-7757, 2006. 
[39] S. K. Cushing, M. Li, F. Huang, and N. Wu, "Origin of strong excitation wavelength dependent fluorescence of graphene oxide," ACS Nano, vol. 8, no. 1, pp. 1002-1013, 2014.

[40] W. Zhang, X. Niu, X. Chen, X. Guo, J. Wang, and J. Fan, "Universal role of oxygen in full-visible-region photoluminescence of diamond nanocrystals," Carbon, vol. 109, pp. 40-48, 2016.

[41] B. Dong, M. Yang, S. Ge, Y. Cao, B. Li, and Y. Lu, "Synthesis and photoluminescence modulating of polypyrrole fluorescent nano-spheres/dots," RSC Advances, vol. 6, no. 28, pp. 2373723745, 2016.

[42] N.-J. Kuo, Y.-S. Chen, C.-W. Wu, C.-Y. Huang, Y.-H. Chan, and I.-W. P. Chen, "One-pot synthesis of hydrophilic and hydrophobic $n$-doped graphene quantum dots via exfoliating and disintegrating graphite flakes," Scientific Reports, vol. 6, article 30426, 2016. 

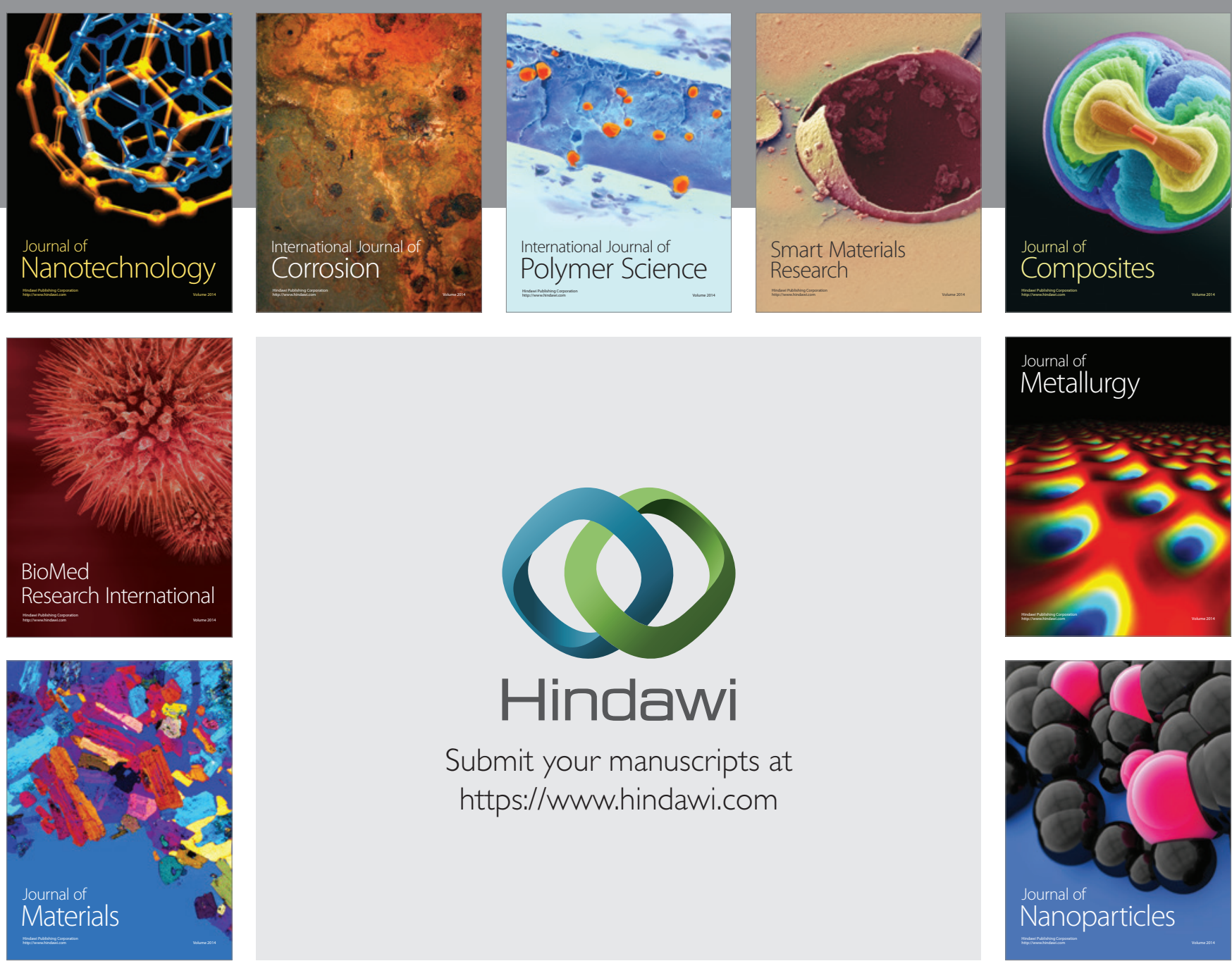

\section{Hindawi}

Submit your manuscripts at

https://www.hindawi.com

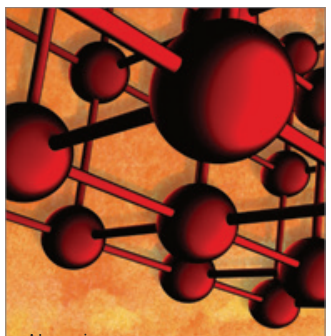

Materials Science and Engineering
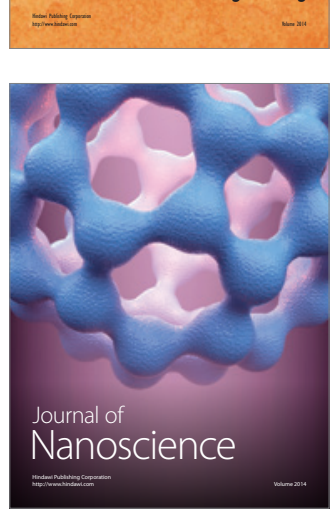
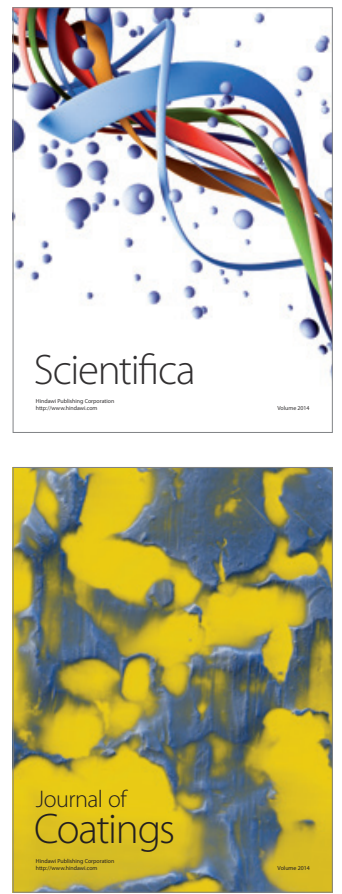
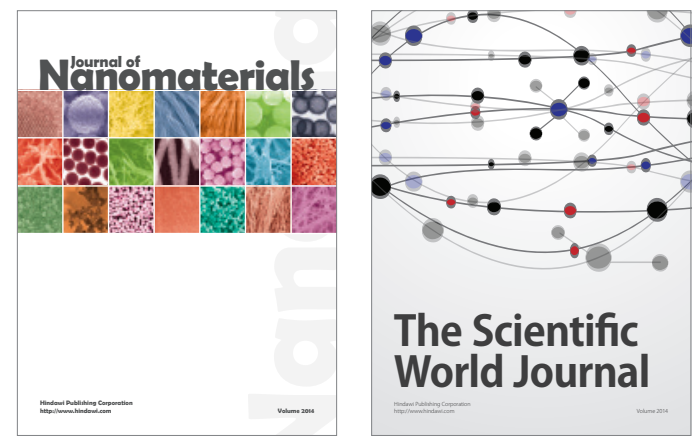

The Scientific World Journal
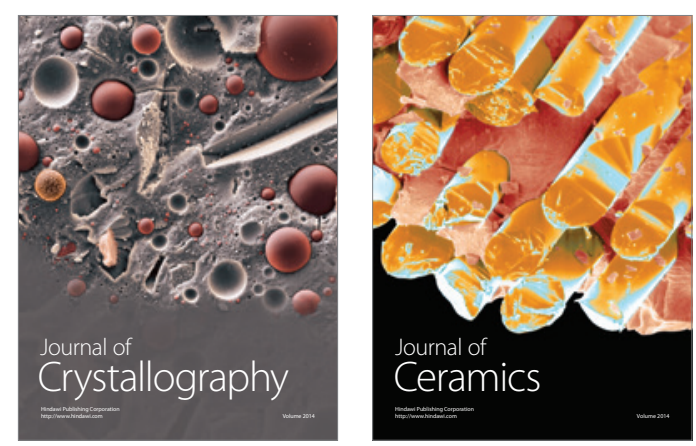
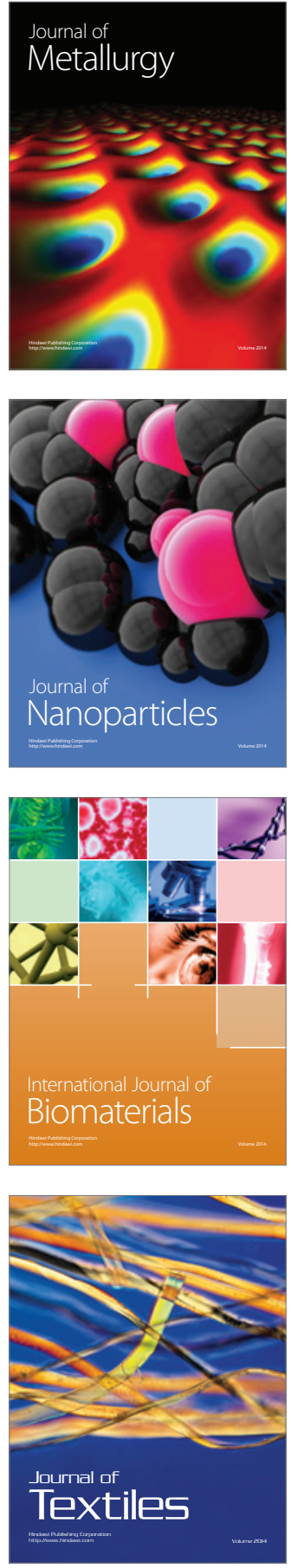